\title{
Can disability predict treatment outcome among traumatized refugees?
}

\author{
Sabrina Friis Jørgensen, Cand.psych.*, Mikkel A. Auning-Hansen, Cand.psych,* Prof. \\ Ask Elklit**
}

\section{Key issues}

- Although the overall level of disability exhibited by traumatized refugees is high and deeply affects their everyday life, it is not a predictor of symptoms of PTSD, anxiety or depression following treatment.

- The ability to 'get along' was a significant predictor of PTSD following treatment.

- A focus on the participants' social relationships is important when planning and providing mental health interventions.

\section{Abstract}

The aim of the present study was to examine the influence of disability on changes in symptoms of PTSD, anxiety, and depression among treatment-seeking traumatized refugees. Eighty-one refugees participated in different rehabilitation programs. PTSD symptomatology was assessed by the HTQ-IV and symptoms of depression and anxiety were assessed by the HSCL-25. Disability was assessed by the WHODAS 2.0 before treatment. Following treatment, no

*) Rehabilitation Center for Torture Survivors - Jutland (RCT-Jutland), Denmark

$\left.{ }^{\star \star}\right)$ National Centre for Psychotraumatology, Institute of Psychology, University of Southern Denmark, Odense, Denmark

Correspondence to: sfjoergensen@rct-jylland.dk statistically significant changes in PTSD, depression, and anxiety symptom scores were observed. Disability in the domain 'getting along' was a significant predictor of PTSD scores following treatment, when controlling for baseline scores. Neither total disability nor individual disability domains predicted any other symptom changes. Living with one's partner did, however, seem to be a consistent and significant predictor of treatment outcome. The results are discussed in terms of clinical implications and future research needs.

Keywords: refugees, disability, PTSD, depression, anxiety, torture survivors

\section{Introduction}

Many refugees have experienced physical and psychological traumas before or during their flight (Kirmayer, et al., 2011). In the post-migration phase, refugees often meet substantial challenges (ACPMH, 2007; Murray, Davidson, \& Schweitzer, 2010). Adverse experiences such as discrimination and acculturation difficulties are common, requiring higher levels of functioning than many refugees have (ACPMH, 2007; Davidson, Murray \& Schweitzer, 2008; Slobodin \& de Jong, 2015; Sachs, 2011). By virtue of their traumatic and adverse experiences, refugees have an elevated risk of developing physical, social, and psychological 
problems (Davidson et al., 2008; Kirmayer, et al., 2011; Nickerson, Bryant, Silove \& Steel, 2011).

Three reviews have examined the efficacy of rehabilitation programs and treatment outcomes (McFarlane \& Kaplan, 2012; Palic \& Elklit, 2011; Slobodin \& de Jong, 2015). The reviews found modest reductions in psychopathological symptoms among refugees. Underlying predictors of treatment outcome remains unknown. There is therefore clearly a need for additional studies examining predictors of treatment outcome in refugee samples to inform rehabilitation programs.

\section{Disability}

Studies have indicated that a lack of treatment effect among traumatized refugees could be due to high levels of symptom severity, disability, and chronicity exhibited by the refugees seeking treatment (Nickerson et al., 2011). Further, other findings have suggested that a diagnosis alone is a poor predictor of treatment outcome (Lund, Sørensen, Christensen, \& Ølholm, 2008; WHO, 2002 \& 2010). Whilst disability has been postulated as a better predictor of treatment outcome than a diagnosis and that high levels of disability may affect treatment efficacy, the influence on treatment outcome among traumatized refugees has yet to be examined. Previous studies have examined the relationship between psychiatric disorders and disability in a unidirectional fashion by conceptualizing disability as the result of symptoms (Rodriguez, Bruce, Pagano \& Keller, 2005). It has been suggested that psychiatric disorders and disability influence and reinforce one another. Ormel et al.

(1994) hypothesized that psychiatric distress could lead to poor psychosocial functioning, which subsequently leads to poorer prognosis or new disease onset. Prospective studies have offered support for a distress-disability cycle hypothesis, by demonstrating that poor psychosocial functioning during remission of anxiety and depression predicted later recurrences (Rodriguez et al., 2005; Solomon et al., 2004). That said, it has not been fully tested if disability can predict treatment outcome in a refugee sample, as tests have only extended to the first link in the distress-disability cycle. On the other hand, several studies have provided evidence that psychiatric disorders are associated with disability among refugees (Buhmann, 2014; Mollica et al., 2001; Momartin, Silove, Manicavasagar, \& Steel, 2004; Sachs, 2011; Steel, Silove, Chey, Bauman, Phan and Phan, 2005; Thapa, Van Ommeren, Sharma, de Jong and Hauff, 2003; Vojvoda, Weine, McGlashan, Becker, \& Southwick, 2008). It is therefore possible that disability might influence the maintenance and severity of PTSD, anxiety, and depression among refugees, and thereby treatment outcome.

Disability is defined as a dysfunction in one or more of the functioning levels (impairments, activity limitations, and participation restrictions) of the International Classification of Functioning (ICF). The ICF can be classified as a bio-psychosocial model, as it takes into account biological, individual, and social perspectives of health, and defines functioning through three levels of human functioning: all body functions, activities, and participation (WHO, 2002). The concept of disability is etiologically neutral, which is useful when assessing disability among refugees experiencing multiple co-occurring physical and psychological conditions (WHO, 2002). An etiologically neutral disability construct makes it possible to examine disability as an independent predictor of changes in symptom levels following treatment. 
Predictors of mental health and treatment outcome There is a general consensus of the impact of pre-migratory experiences on mental health. Several studies have reported a cumulative effect of trauma, where the number of traumatic experiences predicted the severity of psychopathological symptoms (Davidson et al., 2008; Murray et al., 2010). The type of traumatic experiences has also been found to influence symptoms (Davidson et al., 2008; Kirmayer et al., 2011; Steel, Chey, Silove, Marnane, Bryant \& van Ommeren, 2009). However, evidence of the influence of various post-migratory variables on mental health has been inconsistent. These inconsistencies might reflect that the level of distress caused by post-migratory problems depend on several factors, such as, the similarity between the culture of origin and culture of settlement, social support, acceptance by the new nation etc (Buhmann, 2014; Davidson et al., 2008; Kirmayer et al., 2011; Murray et al., 2010; Slobodin and de Jong, 2015). It is therefore likely that results vary across sociocultural contexts. Postmigratory problems also interact with individual characteristics making the salience of various post-migratory problems difficult to evaluate (Davidson et al., 2008; Tempany, 2009). Current daily functioning can be an indicator of the ability to meet the challenges in the post-migration phase, and may represent a more objective indicator of salience for the individual (Sachs, 2011). Studies examining disability as a predictor of treatment outcome might be more easily generalizable across sociocultural contexts.

\section{Study Aims}

The aim of the present study was to examine the influence of disability on changes in symptoms of PTSD, anxiety, and depression among traumatized refugees, when controlling for factors that previous research found to be associated with these psychopathological symptoms. It was hypothesized that disability was a predictor of changes in mental health symptoms among refugees participating in rehabilitation programs.

\section{Methods}

\section{Participants and Design}

The participants were participating in a rehabilitation program in Denmark, having been referred by their general practitioner. The admission criteria for treatment included legal residence, meeting the ICD-10 criteria for PTSD (WHO, 1992), and having experienced a traumatic event in a country outside Denmark. The exclusion criteria included recurring psychotic episodes, substance abuse, and severe needs in terms of stabilization efforts. Patients who had demonstrated at least one baseline measure of symptoms of PTSD, anxiety or depression, and at least one post-treatment measure of the same symptoms, were included in the sample ( $=83$ ) (HTQ IV and the HSCL25). Two patients were removed from the analysis as more than $20 \%$ of the items on the scales measuring PTSD, anxiety, depression, and disability were missing. The number of patients varied in the different analyses as a result of missing data. For example, some of the socio-demographic information was not collected for all patients, and some patients did not answer both questionnaires.

\section{Procedure}

Participants were allocated to different multidisciplinary rehabilitation programs based on the evaluation of their bio-psychosocial resource profile and needs by trained clinicians. They received a mean of 26 treatment sessions over a period of three to six months. The treatment consisted of consultations with psychologists, physiother- 
apists, and social workers. Some participants were also examined by an orthopedic consultant and/or a psychiatrist. The study was conducted in accordance with the Helsinki Declaration and was approved by the Danish Data Protection Agency. Written informed consent was obtained from all participants.

Socio-demographic information was collected during a structured clinical interview prior to obtaining the baseline measurements. Self-report questionnaires were answered pre- and post-treatment. The questionnaires had previously been translated and back-translated into the participants' native languages. If necessary, translation was provided by trained interpreters during assessment and treatment.

\section{Measures}

The socio-demographic information is presented below in Table 2 under Results, and an overview of the treatment variables is presented in Table 3. The remaining data, which came from self-report measures is listed below.

Harvard Trauma Questionnaire-Revised, part IV (HTQ-IV): The HTQ-IV includes 16 items covering PTSD symptoms as described in DSM-IV (Mollica, McDonald, Massagli \& Silove, 2004). Items were scored on a 4-point Likert scale. The cut-off score of $\leq 2.5$ was used in accordance with similar studies on refugee populations (Buhmann, 2014; Palic \& Elkit, 2009; Raghavan, Rasmussen, Rosenfeld, \& Keller, 2013). The reliability estimates in the current study were high with a Cronbach alpha coefficient of .90 at baseline and .96 post-treatment.

The Hopkins Symptom Check List-25 (HSCL-25): The HSCL-25 was used to assess symptoms of depression and anxiety
(Mollica et al., 2004). The HSCL-25 consists of 10 items measuring symptoms of anxiety and 15 items measuring symptoms of depression. Items were scored on a 4-point Likert scale. A cut-off score of $\leq 1.75$ was suggested by the authors and has since been validated in other studies (Lavik, Laake, Hauff, Solberg, 1999; Mollica, Wyshak, de Marneffe, Khuon, \& Lavelle, 1987; Smith Fawzi, Murphy, Pham, Lin, Poole, \& Mollica, 1997). In the current study, the reliability estimates of the HSCL-25 were high at baseline and post-treatment, with Cronbach alpha values of .91 and .96, respectively.

The Brief Pain Inventory - short form (BPI$S F)$ : The BPI-SF consists of two subscales and a total score of pain (Cleeland, 2009; Cleeland \& Ryan, 1994). The BPI-SF consists of four items measuring pain severity at its "worst," "least," "on the average," and "now". Furthermore, seven items measure how much the pain has interfered with seven daily activities. The items were scored on numeric rating scales. For pain intensity, 0 indicated no pain and 10 indicated the worst pain imaginable. For pain interference, 0 indicated no interference and 10 referred to complete interference. The BPI-SF has been demonstrated to have solid psychometric properties across different cultures and clinical conditions (Cleeland, 2009; Cleeland \& Ryan, 1994; Dworkin et al., 2008). The reliability estimate in the current sample was high with a Cronbach alpha coefficient of .94.

\section{The World Health Organization Disability} Assessment Schedule 2.0 (WHODAS 2.0): The WHODAS 2.0 is a 36 items-scale which assesses disability across six domains: cognition, mobility, self-care, getting along, life activities, and participation. In accordance with the guidelines offered by the 
WHO, only 32 items are used if a patient is not currently working or going to school (WHO, 2010). The items were scored on a 5-point Likert scale. In accordance with the WHO's recommendations, domain scores and summary scores were calculated using item-response-theory (IRT) based scoring. Using the IRT-based scoring, the scores were converted into a metric, ranging from 0 (no disability) to 100 (full disability) (WHO, 2010). The WHODAS 2.0 demonstrated good reliability and validity, and a factor structure that has proved consistent across cultures and patient populations (Federici, Meloni, \& Presti, 2009; WHO, 2010). The WHODAS 2.0 defines disability according to the ICF (WHO, 2010). Examples of items in the WHODAS 2.0 can be seen in Table 1 . The reliability ofWHODAS 2.0 (32 items) in the current sample was high with a Cronbach alpha coefficient of .94 .

Table 1: Examples of items in the WHODAS 2.0 (32 items)

\begin{tabular}{|c|c|}
\hline $\begin{array}{l}\text { Domain 1: Cognition } \\
\text { (contains } 6 \text { items) }\end{array}$ & $\begin{array}{l}\text { - In the past } 30 \text { days, how much difficulty did you have in: } \\
\text { "Item 1: Concentrating on doing something for ten minutes? } \\
\text { "Item 3: Analysing and finding solutions to problems in day-to-day life? } \\
\text { "Item 5: Generally understanding what people say }\end{array}$ \\
\hline $\begin{array}{l}\text { Domain 2: Mobility } \\
\text { (contains } 5 \text { items) }\end{array}$ & $\begin{array}{l}\text { - In the past } 30 \text { days, how much difficulty did you have in: } \\
\text { »Item 1: Standing for long periods such as } 30 \text { minutes? } \\
\text { »Item 3: Moving around inside your home? }\end{array}$ \\
\hline $\begin{array}{l}\text { Domain 3: Self-care } \\
\text { (contains } 4 \text { items) }\end{array}$ & $\begin{array}{l}\text { - In the past } 30 \text { days, how much difficulty did you have in: } \\
\text { » Item 1: Washing your whole body? } \\
\text { »Item 3: Eating? }\end{array}$ \\
\hline $\begin{array}{l}\text { Domain 4: Getting along } \\
\text { (contains } 5 \text { items) }\end{array}$ & $\begin{array}{l}\text { In the past } 30 \text { days, how much difficulty did you have in: } \\
\text { »Item 1: Dealing with people you do not know? } \\
\text { »Item 3: Getting along with people who are close to you? }\end{array}$ \\
\hline $\begin{array}{l}\text { Domain 5: Life activities } \\
\text { (contains } 4 \text { items) }\end{array}$ & $\begin{array}{l}\text { - Because of your health condition, in the past } 30 \text { days, how much } \\
\text { difficulty did you have in: } \\
\text { "Item 1: Taking care of your household responsibilities? } \\
\text { "Item 3: Getting all the household work done that you needed to do? }\end{array}$ \\
\hline $\begin{array}{l}\text { Domain 6: Participation } \\
\text { (contains } 8 \text { items) }\end{array}$ & $\begin{array}{l}\text { - In the past } 30 \text { days: } \\
\text { » Item 1: How much of a problem did you have joining in community } \\
\text { activities (for example, festivities, religious or other activities) in the } \\
\text { same way anyone else can? } \\
\text { "Item } 3 \text { : How much of a problem did you have living with dignity } \\
\text { because of the attitudes and actions of others? } \\
\text { "Item } 5 \text { : How much have you been emotionally affected by your health } \\
\text { condition? }\end{array}$ \\
\hline
\end{tabular}




\section{Statistical Analysis}

Statistical analyses were conducted using IBM SPSS Statistic 22. Missing value analyses were performed and Little's MCAR test showed that data missing from the self-report measures was missing completely at random. The expectation maximization technique was used to impute missing values for cases missing less than $20 \%$ values.

Shapiro-Wilk test was used to assess for normal distribution, and the level of significance was set to .05 . Changes in the HTQ-IV scores and the HSCL-25 scores from baseline to post-treatment were evaluated with paired-samples t-test for continuous parametric variables, and Wilcoxon signed-rank test for non-parametric variables. If the assumptions of the Wilcoxon signed-rank tests were violated, exact sign tests were used. Cohen's d was calculated to evaluate the effect sizes of changes in symptoms of PTSD, anxiety, and depression. McNemar's tests were used to determine significant differences in the proportion of participants scoring above cut-off for PTSD, anxiety, and depression at baseline and post treatment.

A zero-order correlation matrix was generated to determine whether there was an association between disability scores and post-treatment symptom scores. Thereafter, significant predictors of treatment outcome $(p<.05)$ were identified in regression models adjusting for the corresponding baseline score. Potential predictors included socio-demographic variables (Table 2), treatment variables (Table 3), pain severity and pain interference (Table 4), baseline HSCL-25 and HTQ-IV scores (Table 5), and WHODAS 2.0 scores (Table 4).

Significant predictors were subsequently included in multiple regression analyses. The post treatment HTQ-IV PTSD model included the following predictors: baseline HTQ-IV PTSD scores, WHODAS domain 'getting along', age, living with one's partner, and being single. The post-treatment HSCL-25 depression model included baseline HSCL-25 depression scores, living with one's partner, and BPI interference scores. Finally, the post treatment HSCL-25 anxiety model included baseline HSCL-25 anxiety scores, orthopedic consultation, living with one's partner, being single, BPI severity, and BPI interference.

\section{Results}

The current sample spanned 18 nationalities, including a majority from Bosnia (30\%), Afghanistan (12\%), and Syria (11\%), respectively. Nearly $52 \%$ of the sample was female with a mean age of 44.20 years. The majority of the sample was married $(72.8 \%)$. The mean number of years living in Denmark was 15.

An overview of treatment sessions is presented in Table $3.32 \%$ participants participated in at least one treatment session with the psychiatrist, and 56 received psycho-pharmacological medicine. There were more participants receiving psychopharmacological medicine than consultations with the psychiatrist. These numbers reflect that some participants were treated with psycho-pharmacological medicine by their general practitioner or a psychiatrist elsewhere.

Table 4 displays mean scores for pain and disability measures. $99 \%$ of the participants reported having experienced pain within the last 24 hours. 65 participants answered 32 WHODAS items, but only 25 participants answered all 36 items on the WHODAS 2.0. Therefore, only the 32 items were included in the analyses. 
Table 2: Overview of socio-demographic variables $(N=81)$

\begin{tabular}{|c|c|c|c|}
\hline$(\mathrm{N}=81)$ & $\mathrm{n} / \mathrm{m}$ & $(\mathrm{SD}) \%$ & Range \\
\hline Age & 44.20 & $(9.5)$ & $23-62$ \\
\hline Male & 39 & $48.1 \%$ & - \\
\hline Female & 42 & $51.9 \%$ & - \\
\hline Mean years in Denmark & 15.1 & $(7.5)$ & $1-31$ \\
\hline Danish citizenship [n=81] & 23 & $28.4 \%$ & - \\
\hline Need for interpreter $[\mathrm{n}=81]$ & 52 & $64.2 \%$ & - \\
\hline Mean years in school in country of origin & 10.3 & $(3.6)$ & $0-17$ \\
\hline Education in country of origin $[\mathrm{n}=57]$ & 37 & $64.9 \%$ & - \\
\hline \multicolumn{4}{|l|}{ Family in country of origin: } \\
\hline Parents [n=65] & 40 & $61.5 \%$ & - \\
\hline Partner/spouse $[\mathrm{n}=60]$ & 2 & $3.3 \%$ & - \\
\hline Siblings $[n=68]$ & 53 & $77.9 \%$ & - \\
\hline Children $u 18$ years $[\mathrm{n}=56]$ & 3 & $5.4 \%$ & - \\
\hline Children $18+$ years $[\mathrm{n}=57]$ & 5 & $8.8 \%$ & - \\
\hline \multicolumn{4}{|l|}{ Marital status: } \\
\hline Married & 59 & $72.8 \%$ & - \\
\hline Single & 9 & $11.1 \%$ & - \\
\hline Divorced/ Separated & 11 & $13.6 \%$ & - \\
\hline Widowed & 2 & $2.5 \%$ & - \\
\hline \multicolumn{4}{|l|}{ Income: } \\
\hline Salary & 13 & $16 \%$ & - \\
\hline Social Security benefit & 41 & $50.6 \%$ & - \\
\hline Jobseeker's allowance & 3 & $3.7 \%$ & - \\
\hline Disability pension & 5 & $6.2 \%$ & - \\
\hline Sickness benefits & 17 & $21 \%$ & - \\
\hline Other & 2 & $2.5 \%$ & - \\
\hline Worked in DK [n=66] & 51 & $77.3 \%$ & - \\
\hline Worked in country of origin $[\mathrm{n}=59]$ & 47 & $79.7 \%$ & - \\
\hline \multicolumn{4}{|l|}{ Social network: } \\
\hline Family members outside primary family $[\mathrm{n}=70]$ & 44 & $62 \%$ & - \\
\hline Friends with other ethnicity than Danish $[n=70]$ & 49 & $70 \%$ & - \\
\hline Danish friends $[n=60]$ & 40 & $66.7 \%$ & - \\
\hline Living with partner $[\mathrm{n}=61]$ & 52 & $85.2 \%$ & - \\
\hline
\end{tabular}


Table 3: Overview of treatment variables

\begin{tabular}{llll}
\hline$(\mathrm{N}=81)$ & $\mathrm{n} / \mathrm{m}$ & $(\mathrm{SD}) \%$ & Range \\
\hline Individual treatment sessions & 25.6 & $(11.3)$ & $4-51$ \\
Psychotherapy sessions & 9.9 & $(4.3)$ & $0-30$ \\
Social work sessions & 7.8 & $(5.2)$ & $0-28$ \\
Physiotherapy sessions & 7.7 & $(4.8)$ & $0-20$ \\
Consultation with the orthopedic consultant & 54 & $66.7 \%$ & - \\
$\begin{array}{l}\text { Consultation with the psychiatrist } \\
1 \text { type of psycho-pharmacological medicine during } \\
\text { treatment }\end{array}$ & 35 & $43.2 \%$ & - \\
$\begin{array}{l}\text { > } 1 \text { type of psychopharmacological medicine during } \\
\text { treatment }\end{array}$ & 33 & $69.1 \%$ & - \\
\hline
\end{tabular}

Table 4: Descriptive statistics for pain and disability

\begin{tabular}{|c|c|c|c|c|c|}
\hline \multirow{2}{*}{$\begin{array}{l}(\text { WHODAS } 2.0: \mathrm{N}=65) \\
(\mathrm{BPI}-\mathrm{SF}: \mathrm{N}=77)\end{array}$} & \multirow{2}{*}{ M } & \multirow{2}{*}{ (SD) } & \multirow{2}{*}{ Range } & \multicolumn{2}{|c|}{ Disability $^{\mathrm{a}}$} \\
\hline & & & & $\mathrm{N}$ & $\%$ \\
\hline BPI-SF total & 7.1 & $(2.1)$ & $.36-9,8$ & & \\
\hline BPI-SF Pain severity & 6.6 & $(2.2)$ & $0-10$ & & \\
\hline BPI-SF Pain interference & 7.4 & (2.3) & $0-10$ & & \\
\hline WHODAS Total disability & 65.1 & (20) & $16.9-95.3$ & 65 & 100 \\
\hline WHODAS Cognition & 68.5 & $(22.7)$ & $0-100$ & 64 & 98.5 \\
\hline WHODAS Mobility & 63.6 & $(26.7)$ & $0-100$ & 63 & 96.9 \\
\hline WHODAS Self-care & 46 & $(26.5)$ & $0-90$ & 60 & 92.3 \\
\hline WHODAS Getting along & 66.3 & $(30.2)$ & $0-100$ & 63 & 96.9 \\
\hline WHODAS Life activities & 71.8 & $(29.1)$ & $0-100$ & 63 & 96.9 \\
\hline WHODAS Participation & 67.6 & $(20.8)$ & $13.7-100$ & 65 & 100 \\
\hline
\end{tabular}

Note: a Summary score or domain score above 0 .

WHODAS $=$ World Health Organization Disability Assessment Schedule 2.0

$\mathrm{BPI}-\mathrm{SF}=$ the Brief Pain Inventory - short form 
Table 5: Changes in symptoms of anxiety, depression, and PTSD

\begin{tabular}{|c|c|c|c|c|c|c|c|c|c|c|c|}
\hline \multirow{2}{*}{$\begin{array}{l}(\text { HSCL-25: } \mathrm{N}=75) \\
(\text { HTQ-IV: } \mathrm{N}=69)\end{array}$} & \multicolumn{3}{|c|}{ Baseline } & \multicolumn{3}{|c|}{ Post treatment } & \multicolumn{3}{|c|}{ Diff. score } & & \multirow{2}{*}{$d$} \\
\hline & $M$ & (SD) & Range & M & (SD) & Range & $M$ & (SD) & Range & & \\
\hline \multirow[t]{2}{*}{ HSCL-25 Total } & 7.1 & $(.54)$ & $1.40-$ & 2.97 & (.66) & $1.20-$ & .11 & $(.59)$ & -1.1 & $.49^{\mathrm{a}}$ & .21 \\
\hline & & & 3.87 & & & 3.82 & & & -2.2 & & \\
\hline \multirow[t]{2}{*}{ HSCL-25 Anxiety } & 6.6 & $(.59)$ & $1.60-4$ & 2.98 & $(.72)$ & $1-4$ & .14 & $(.67)$ & -1.1 & $.20^{\mathrm{a}}$ & .16 \\
\hline & & & & & & & & & -2.1 & & \\
\hline \multirow{2}{*}{$\begin{array}{l}\text { HSCL-25 } \\
\text { Depression }\end{array}$} & 7.4 & $(.56)$ & $1.27-$ & 2.97 & $(.66)$ & $1.27-$ & .10 & $(.61)$ & -1.1 & $.38^{\mathrm{a}}$ & .16 \\
\hline & & & 3.87 & & & 3.90 & & & -2.3 & & \\
\hline \multirow{2}{*}{ HTQ-IV PTSD } & 65.1 & (.49) & $1.56-$ & 3.05 & $(.65)$ & $1.25-4$ & .09 & $(.65)$ & -1.63 & $.47^{\mathrm{a}}$ & .13 \\
\hline & & & 3.88 & & & & & & -2.44 & & \\
\hline $\begin{array}{l}\text { HTQ- IV Re- } \\
\text { experiencing }\end{array}$ & 68.5 & $(2.58)$ & $6-16$ & 12.78 & (2.93) & $\begin{array}{l}5.84- \\
16\end{array}$ & .37 & (3.12) & $-7-10$ & $.70^{\mathrm{b}}$ & .19 \\
\hline HTQ-IV Avoidance & 63.6 & $(3.77)$ & $9-27$ & 20.05 & $(4.44)$ & $9-28$ & .77 & $(4.67)$ & $-1-15$ & $.18^{\mathrm{c}}$ & .08 \\
\hline HTQ-IV Arousal & 46 & $(2.76)$ & $9-20$ & 16.02 & (3.62) & $5-20$ & .26 & (3.98) & $-9-14$ & $.70^{\mathrm{b}}$ & 92.3 \\
\hline
\end{tabular}

Note: ${ }^{a}$ Wilcoxon signed-rank test; ${ }^{b}$ Sign test; 'Paired-samples t-test., HTQ-IV = Harvard Trauma QuestionnaireRevised, part IV, HSCL-25 = The Hopkins Symptom Check List-25

Changes in Symptom Scores

An overview of the HSCL-25 scores, HTQ-IV scores, differential scores, p-values and Cohen's d are presented in Table 5.74 out of 75 participants $(98.7 \%)$ scored above the cut-off score of $\leq 1.75$ on the anxiety and depression subscales at baseline. Post-treatment, 5 (6.7\%) participants scored below cut-off for depression and 7 (9.3\%) for anxiety. Using exact McNemar's tests, this decrease in participants scoring clinically significant symptoms was not statistically significant. Effect sizes were small for all the changes in symptom scores $(d=.16-.21)$.

At baseline, 60 out of 69 participants ( 87 $\%$ ) scored above the cut-off score of $\leq 2.5$ for PTSD which decreased to 56 (81\%) post-treatment. An exact McNemar's test revealed that this decrease was not statistically significant $(p=.42)$. Effect sizes were small for all the changes in the HTQ-IV subscale scores $(d=.08-.19)$.

Predictors of Change

A zero-order correlation matrix is presented in
Table 6. The results of the multiple regression analyses for each outcome are presented in Table 7. The multiple regression model predicting post-treatment PTSD scores was statistically significant $[F(5,36)=9.18, p<$ .001 , adj. $R^{2}=.50$ ] with 'living with one's partner' and the WHODAS 2.0 domain 'getting along' significantly adding to the prediction. In the preliminary regression analyses, having parents, a partner, or children under the age of 18 in the country of origin were also identified as significant predictors of post treatment PTSD scores. However, in the PTSD regression model, variables regarding family in the country of origin were excluded, as there was no data available about these variables for 16 to 25 participants.

The multiple regression model predicting post-treatment depression was also significant $\left[F(3,50)=17.48, p<.001\right.$, adj. $\left.R^{2}=.48\right]$. Baseline anxiety scores and living with one's partner proving to be significant predictors.

The regression model predicting posttreatment anxiety was also statistically significant $\left[F(6,46)=7.54, \mathrm{p}<.001\right.$, adj. $R^{2}$ 
$=.43]$ with baseline anxiety scores and living with one's partner adding significantly to the prediction. Being married was also a significant predictor in the preliminary regression analyses, however, it was dropped from the anxiety regression model, as its correlation with the variable 'being single' was an issue concerning multicollinearity.

\section{Discussion}

The findings indicated that participants overall exhibited high levels of disability at baseline. Comparing the disability scores with population norms provided by the WHO(2010), the mean disability score was above the 90th population percentile. Other studies also reported high levels of disability among refugees compared to the general population and psychiatric in-patients (Palic,
Kappel, Nielsen, Carlsson, \& Bech, 2014; Steel et al., 2005). The number of participants reporting pain in the current sample was also very high (99\%), yet similar to other studies of traumatized refugees in clinical settings (Buhmann, 2014). Findings indicated that baseline PTSD, depression, and anxiety scores were high and consistent with other studies of traumatized refugees in clinical settings (Buhmann, 2014; Palic \& Elkit, 2009; Carlsson, Olsen, Mortensen, \& Kastrup, 2006; Carlson, Mortensen, \& Kastrup, 2005).

Although the HSCL-25 scores and the HTQ-IV scores improved following treatment, the level of change was non-significant. Previous studies have reported similar non-significant reductions in overall levels of PTSD, anxiety, and depression (Birck, 2001; Carlsson et al., 2005; Mollica, Wyshak,

Table 6: Zero-order correlation matrix between disability scores and pre- and post-symptom scores

\begin{tabular}{|c|c|c|c|c|c|c|}
\hline & $\begin{array}{l}\text { Baseline } \\
\text { HTQ IV } \\
\text { PTSD } \\
(\mathrm{N}=59)\end{array}$ & $\begin{array}{l}\text { Post } \\
\text { treatment } \\
\text { HTQ IV } \\
\text { PTSD } \\
(\mathrm{N}=59)\end{array}$ & $\begin{array}{l}\text { Baseline } \\
\text { HSCL-25 } \\
\text { anxiety } \\
(\mathrm{N}=63)\end{array}$ & $\begin{array}{l}\text { Post } \\
\text { treatment } \\
\text { HSCL- } \\
\text { 25anxiety } \\
(\mathrm{N}=63)\end{array}$ & $\begin{array}{l}\text { Baseline } \\
\text { HSCL-25 } \\
\text { depres- } \\
\text { sion } \\
(\mathrm{N}=63)\end{array}$ & $\begin{array}{l}\text { Post } \\
\text { treatment } \\
\text { HSCL- } \\
25 \text { depres- } \\
\text { sion } \\
(\mathrm{N}=63)\end{array}$ \\
\hline WHODAS Total disability & $.733^{\star \star}$ & $.409^{\star \star}$ & $.600^{\star \star}$ & $.302^{\star}$ & $.640^{\star \star}$ & $.464^{\star \star}$ \\
\hline WHODAS Cognition & $.580^{\star \star}$ & $.259^{\star}$ & $.470^{\star \star}$ & .171 & $.483^{\star \star}$ & $.332^{\star \star}$ \\
\hline WHODAS Mobility & $.662^{\star \star}$ & $.384^{\star \star}$ & $.451^{\star \star}$ & .220 & $.521^{\star \star}$ & $.381^{\star \star}$ \\
\hline WHODAS Self-care & $.422^{\star \star}$ & .170 & $.429^{\star \star}$ & .099 & $.393^{\star \star}$ & .215 \\
\hline WHODAS Getting along & $.629^{\star \star}$ & $.465^{\star \star}$ & $.520^{\star \star}$ & $.345^{\star \star}$ & $.570^{\star \star}$ & $.481^{\star \star}$ \\
\hline WHODAS Life activities & $.591^{\star \star}$ & $.348^{\star \star}$ & $.441^{\star \star}$ & $.250^{\star}$ & $.417^{\star \star}$ & $.266^{\star}$ \\
\hline WHODAS Participation & $.617^{\star \star}$ & $.337^{\star \star}$ & $.500^{\star \star}$ & $.300^{\star \star}$ & $.569^{\star \star}$ & .433 \\
\hline
\end{tabular}

${ }^{\star} p<.05 ;{ }^{\star \star} p<.005$ 
Table 7: Summary of multiple regression analyses examining post treatment HSCL-25 scores and HTQ-IV scores

\begin{tabular}{|c|c|c|c|c|}
\hline Variable & B & $\mathrm{SE}_{\mathrm{B}}$ & $\beta$ & Adj. $R^{2}$ \\
\hline $\begin{array}{l}\text { Dependent variable: Post treatment HSCL-25 anxiety } \\
\text { scores }(\mathrm{N}=53)\end{array}$ & & & & .43 \\
\hline BPI-SF total & .40 & .12 & $.40^{\star \star}$ & \\
\hline BPI-SF Pain severity & .04 & .15 & .03 & \\
\hline BPI-SF Pain interference & .43 & .20 & $.28^{\star}$ & \\
\hline WHODAS Total disability & .03 & .04 & .1 & \\
\hline WHODAS Cognition & .41 & .79 & .18 & \\
\hline WHODAS Mobility & -.23 & .26 & -.12 & \\
\hline $\begin{array}{l}\text { Dependent variable: Post treatment HSCL-25 } \\
\text { depression scores }(\mathrm{N}=54)\end{array}$ & & & & .48 \\
\hline Baseline HSCL-25 depression & .60 & .13 & $.54^{\star \star}$ & \\
\hline Living with partner & .52 & .16 & $.32^{\star \star}$ & \\
\hline BPI interference & .06 & .03 & .21 & \\
\hline $\begin{array}{l}\text { Dependent variable: Post treatment HTQ-IV PTSD } \\
\text { scores }(\mathrm{N}=42)\end{array}$ & & & & .50 \\
\hline Baseline HTQ-IV PTSD & .26 & .16 & .26 & \\
\hline WHODAS 'getting along' & .01 & .003 & $.39^{\star}$ & \\
\hline Age & .01 & .01 & .11 & \\
\hline Living with partner & .62 & .30 & $.40^{\star}$ & \\
\hline Being single & .05 & .37 & .03 & \\
\hline
\end{tabular}

Note: ${ }^{\star} p<.05 ;{ }^{\star \star} p<.01$

Lavelle, Truong, Tor, \& Yang, 1990), while others found statistically significant symptom reductions following multidisciplinary treatment (Brune, Haasen, Krausz, Yagdiran, Bustos, \& Eisenman, 2002; Carlsson et al., 2005; Raghavan et al., 2013). There may be several reasons for these non-significant findings. First, the participants had been living in Denmark for a mean of 15 years, indicating that their traumatic experiences occurred many years ago. It was therefore plausible that trauma-related distress may have been present for several years and possibly chronic in nature. Similar studies have also reported long-lasting distress levels that seemed to be chronic (Birck, 2001; Carlsson et al., 2005; Palic \& Elkit, 2009). Second, it has been suggested that participants admitted to specialized treatment centers comprise some of the most severely affected refugees (Carlsson et al., 2005; Nickerson et al., 2011), and prolonged absence of treatment possibly had a negative effect on distress levels over time (Nickerson et al., 2011). In the present study, no single treatment-related variables predicted treatment outcome, indicating that traumatized refugees represent a group of participants which is somewhat resistant to treatment. Importantly, however, other studies 
have argued treatment effect is not typically evident until six months after the end of treatment (Lund et al., 2008). It may therefore be too soon to draw any conclusions regarding the efficacy of the rehabilitation programs in treating PTSD, anxiety, and depression. The RCT-Jutland is currently preparing a nine-month follow-up study. Further analyses of follow-up data might reveal whether there is any long-lasting or a delayed treatment effect.

\section{Predictors of treatment outcome}

Overall, few predictors of treatment outcome were found which might have been the result of methodological issues. Nevertheless, it was hypothesized that disability would be a statistically significant predictor of changes in symptoms of PTSD, anxiety, and depression following treatment. Disability in the domain of 'getting along' was a statistically significant predictor for post-treatment PTSD scores. In fact, higher levels of disability within the domain of 'getting along' predicted higher PTSD scores following treatment. 'Living with one's partner' was also a significant predictor with respect to PTSD and was associated with higher symptom scores following treatment. In similar studies, social support has conversely often been found to be a protective factor for the development and maintenance of psychopathological symptoms among refugees (Davidson et al., 2008; Kirmayer et al., 2011). On the other hand, stressors in family life have been found to negatively impact symptom severity and treatment effect of PTSD (Lund et al., 2008). Anger, marital conflicts, and domestic violence are common problems among refugees suffering from PTSD, often making PTSD symptoms more sever (ACPMH, 2007; Hinton, Rasmussen, Nou, Pollack, \& Mary-Jo, 2009; Nilsson, Brown, Russell, \& Khamphakdy-Brown, 2008). In this study, 'living with one's partner' also predicted higher post-treatment anxiety and depression scores. As 96 percent of the participants experienced disability in the domain of 'getting along', it is perhaps not surprising that 'living with one's partner' was associated with poorer treatment outcome.

Neither total disability nor individual disability domains predicted any other symptom changes. There are several possible explanations for this finding. Firstly, disability might not be a predictor of treatment outcome as hypothesized. It has been suggested that it is necessary for refugees to address trauma-related symptoms before being able to manage other psychosocial stressors (Nickerson et al., 2011). According to this argument, disability can be seen as a consequence of trauma-related symptoms and, in order to reduce disability, the trauma-related symptoms would have to be treated first (Nickerson et al., 2011).

Secondly, whilst disability has been postulated as a better predictor of treatment outcome than a diagnosis among other populations, there are, however, some substantial differences in disability exhibited by refugees and other treatment-seeking populations. These differences include the severity of disability and magnitude of change in disability scores following treatment (Buhmann, 2014; Palic et al., 2014; Steel et al., 2005; Tempany, 2009).

Even though the results indicated that disability was not a predictor of treatment outcome, the methodological issues suggest that caution should be used in drawing any definitive conclusions. For example, the small sample size may increase the risk of type II errors and complicate generalization. The sample was also quite homogeneous regarding the level of mental health symptoms, which could also lead to type II errors. 


\section{Implications}

The level of disability exhibited by the participants was very high and affected several life domains, suggesting that disability should still be a focus of rehabilitation. Generally, the results suggested that the participants faced multiple co-occurring and complex problems, indicating a need for multidisciplinary treatment. However, if the aim of treatment is solely to reduce symptoms of PTSD, anxiety, and depression, it is questionable whether participants should be allocated to different treatment programs based on their level of functioning, rather than the severity of psychopathological symptoms. It is recommended that future studies examining disability among refugees should also include culture-sensitive measures of disability to gain an insight into the disability experienced by the individual and to see whether a culture-sensitive measure of disability might predict treatment outcome.

The finding that disability in the domain of 'getting along' predicted less improvement in symptoms of PTSD, and that 'living with one's partner' predicted less overall symptom improvement indicates that a focus on the participants' social relationships is important when planning and providing mental health interventions. Interventions would benefit from including the family of the patient, especially the partner, if the patient wishes to do so.

\section{Limitations}

The present study has important limitations. Firstly, participants were allocated to different treatment programs, but considered as one sample. Despite this heterogeneity, all were traumatized, participating in treatment at the same treatment center, and receiving treatment based on the rehabilitation perspective. Secondly, the study design was not randomized nor controlled, which means that causal conclusions regarding the relationship between predictor variables and treatment outcome must be drawn with caution. However, the study was naturalistic and exploited clinical data and as such it has high external generalizability. Thirdly, self-report questionnaires were used. When using self-report questionnaires, the risk of reporting bias increases. Steel et al. (2009) found a higher prevalence of mental disorders when using self-report questionnaires. Nevertheless, the rates of symptoms of PTSD, depression, and anxiety in the present study were similar to other comparable studies (Buhmann, 2014; Palic \& Elkit, 2009). Finally, change in psychopathological symptoms is not necessarily a fully encapsulating outcome, as they do not embrace rehabilitation in its wider, holistic sense (Johansen, Rahbek, Møller, \& Jensen, 2004). It is possible that the inclusion of other outcome measures would have contributed to a better understanding of the impact of disability.

\section{Conclusion}

The refugees referred to treatment at the RCT-Jutland exhibited high levels of pain, disability, and symptoms of PTSD, anxiety, and depression, indicating a crucial need for treatment in this particular population.

Following treatment, no statistically significant changes in symptom scores were observed.

With respect to PTSD, disability in the domain of 'getting along' was a significant predictor of post-treatment scores. Other regression analyses found that neither global disability scores nor the disability domains predicted other treatment outcomes.

Generally, few variables seemed to predict treatment outcome, which could be due to methodological limitations. 'Living with one's partner' did however seem to be a consistent and significant predictor of treatment outcome, which indicates the importance of 
including the family of the patient in treatment, whenever possible.

\section{References}

Australian Centre For Posttraumatic Mental Health (ACPMH) (2007). Australian Guideline for the treatment of Adults with Acute Stress Disorder and Posttraumatic Stress Disorder. Melbourne: ACPMH.

Birck A. (2001). Torture victims after psychotherapy - a two year follow-up. Torture, 11(2), 55-58.

Brune, M, Haasen, C, Krausz, M, Yagdiran, O, Bustos, E, Eisenman, D. (2002). Belief systems as coping factors for traumatized refugees: a pilot study. European Psychiatry. 17: 451-58.

Buhmann, CB. (2014). Traumatized refugees: Morbidity, treatment and predictors of outcome. Danish Medical Journal, 61(8): B4871.

Carlsson, JM, Mortensen, EL, Kastrup, M. (2005). A Follow-Up Study of Mental Health and Health-Related Quality of Life in Tortured Refugees in Multidisciplinary Treatment. The Journal of Nervous and Mental Disease, 19 (10): 651-57

Carlsson, JM, Olsen, JM, Mortensen, EL, Kastrup, M. (2006). Mental Health and Health-Related Quality of Life A 10-Year Follow-Up of Tortured Refugees. The Journal of Nervous and Mental Disease, 194 (10): 725-31.

Cleeland, CS. (2009). The Brief Pain Inventory User Guide. Retrieved from https://www.mdanderson.org/education-and-research/departmentsprograms-and-labs/departments-and-divisions/ symptom-research/symptom-assessment-tools/ brief-pain-inventory-users-guide.html .

Cleeland, CS, Ryan, KM. (1994). Pain assessment: Global use of the brief pain inventory. Annals of the Academy of Medicine, Singapore, 23 (2): 129-38.

Davidson, GR, Murray, KE, Schweitzer, R. (2008). Review of refugee mental health and wellbeing: Australian perspectives. Australian Psychologist, 43 (3), 160-74

Dworkin, RH, Turk, DC, Wyrwich, KW, Beaton D, Cleeland, CS, Farrar, JT et al. (2008). Interpreting the Clinical Importance of Treatment Outcomes in Chronic Pain Clinical Trials: IMMPACT Recommendations. The Journal of Pain, 9 (2): 105-21

Federici, S, Meloni, F, Presti, AL. (2009). International Literature Review on WHODAS II (World Health Organization Disability Assessment Schedule II). Life Span and Disability, 12 (1): 83-110.
Hinton D, Rasmussen A, Nou L, Pollack M, MaryJo G. (2009). Anger, PTSD, and the Nuclear Family: A Study of Cambodian Refugees. Social Science \& Medicine, 69 (9): 1387-94.

Johansen, JS, Rahbek, J, Møller, K, Jensen, L. (2004). Hvidbog om rehabiliteringsbegrebet. Århus: MarselisborgCentret.

Kirmayer, LJ, Narasiah, L, Munoz, M, Rashid, M, Ryder, AG, Guzder, J et al. (2011). Canadian Guidelines for Immigrant Health: Common mental health problems in immigrants and refugees: general approach in primary care. Canadian Medical Association Journal, 183 (12): 959-67.

Lavik, NJ, Laake, P, Hauff, E, Solberg, Ø. (1999). The use of self-reports in psychiatric studies of traumatized refugees: Validation and analysis of HSCL-25. Nordic Journal of Psychiatry, 53 (1): 17-20.

Lund M, Sørensen JH, Christensen JB, Ølholm A. (2008). MTV om behandling og rehabilitering af PTSD - herunder traumatiserede flygtninge. Vejle: La Cour Offset.

McFarlane CA, Kaplan I. (2012). Evidence-based psychological interventions for adult survivors of torture and trauma: A 30-year review. Transcultural Psychiatry, 49 (3-4): 539-67.

Mollica, RF, McDonald, LS, Massagli, MP, Silove DM. (2004). Measuring trauma, measuring torture: instructions and guidance on the utilization of the Harvard Program in Refugee Trauma's Versions of the Hopkins Symptom Checklist-25 (HSCL-25) and the Harvard Trauma Questionnaire (HTQ). Cambridge, MA: Harvard Program in Refugee Trauma.

Mollica, RF, Sarajlic, N, Chernoff, M, Lavelle, J, Vukovic, IS, Massagli, MP. (2001). Longitudinal study of psychiatric symptoms, disability, mortality, and emigration among Bosnian refugees. JAMA, 286 (5): 546-54.

Mollica, RF, Wyshak, G, Lavelle, J, Truong, T, Tor, S, Yang, T. (1990). Assessing symptom change in Southeast Asian refugee survivors of mass violence and torture. The American Journal of Psychiatry, 147 (1): 83-88.

Mollica, RF, Wyshak, G, de Marneffe, D, Khuon, F, Lavelle, J. (1987). Indochinese versions of the Hopkins Symptom Checklist-25: a screening instrument for the psychiatric care of refugees. The American Journal of Psychiatry, 144 (4): 497-500.

Momartin, S, Silove, D, Manicavasagar, V, Steel, Z. (2004). Comorbidity of PTSD and depression: associations with trauma exposure, symptom severity and functional impairment in Bosnian 
refugees resettled in Australia. Journal of Affective Disorders, 80: 231-38.

Murray, KE, Davidson, GR, Schweitzer, RD. (2010). Review of Refugee Mental Health Interventions Following Resettlement: Best Practices and Recommendations. American Journal of Orthopsychiatry, 80 (4): 576-85.

Nickerson, A, Bryant, RA, Silove, D, Steel, Z. (2011). A critical review of psychological treatments of posttraumatic stress disorder in refugees. Clinical Psychology Review, 31: 399-417.

Nilsson, JE, Brown, C, Russell, EB, KhamphakdyBrown, S. (2008). Acculturation, Partner Violence, and Psychological Distress in Refugee Women From Somalia. Journal of Interpersonal Violence, 23 (111): 1654-63.

Ormel, J, VonKorff, M, Ustun, TB, Pini, S, Korten, A, Oldehinkle, T. (1994). Common Mental disorders and disability across cultures: Results from WHO Collaborative Study of Psychological Problems in General Health Care. JAMA, 272: 1741-48.

Palic, S, Elklit, A. (2009). An explorative outcome study of CBT-based multidisciplinary treatment in a diverse group of refugees from a Danish treatment centre for rehabilitation of traumatized refugees. Torture, 19 (3): 248-70.

Palic, S, Elklit, A. (2011). Psychosocial treatment of posttraumatic stress disorder in adult refugees: a systematic review of prospective treatment outcome studies and a critique. Journal of Affective Disorders, 131 (1-3): 8-23.

Palic, S, Kappel, LM, Nielsen MS, Carlsson, J, Bech, P. (2014). Comparison of psychiatric disability on the health of nation outcome scales (HoNOS) in resettled traumatized refugee outparticipants and Danish inparticipants. BMC Psychiatry, 14: 330-40.

Raghavan, S, Rasmussen, A, Rosenfeld, B, Keller, AS. (2013). Correlates of symptom reduction in treatment-seeking survivors of torture. Psychological Trauma: Theory, Research, Practice, and Policy, 5 (4): 377-83.

Rodriguez, BF, Bruce, SE, Pagano, ME, Keller, MB. (2005). Relationships among psychosocial functioning, diagnostic comorbidity, and the recurrence of generalized anxiety disorder, panic disorder, and major depression. Journal of Anxiety disorders, 19: 752-66.

Sachs, E. (2011). Assessing the Impact of Psychological Distress on the Daily Functioning of Refugees: Creating a High-Risk Symptom Profile for Disability among Nonwestern Trauma Survivors. New York: UMI Dissertation Publishing.

Slobodin, O, de Jong, JTVM. (2015). Mental health interventions for traumatized asylum seekers and refugees: What do we know about their efficacy? International Journal of Social Psychiatry, 61 (1): 17-26.

Smith Fawzi MC, Murphy E, Pham T, Lin L, Poole C, Mollica RF. (1997). The validity of screening for post-traumatic stress disorder and major depression among Vietnamese former political prisoners. Acta Psychiatrica Scandinavica, 95 (2): 87-93.

Solomon DA, Leon AC, Endicott J, Mueller TI, Coryell W, Shea T, Keller MB. (2004). Psychosocial impairment and recurrence of major depression. Comprehensive Psychiatry, 45 (6): 423-30.

Steel, Z, Chey, T, Silove, D, Marnane, C, Bryant, RA, van Ommeren, M. (2009). Association of torture and other potentially traumatic events with mental health outcomes among populations exposed to mass conflict and displacement: A systematic review and meta-analysis. Journal of the American Medical Academy, 302 (5): 537-49.

Steel, Z, Silove, D, Chey, T, Bauman, A, Phan, T, Phan, T. (2005) Mental disorders, disability and health service use amongst Vietnamese refugees and the host Australian population. Acta Psychiatrica Scandinavica, 111:300-309.

Tempany, M. (2009). What Research tells us about the Mental Health and Psychosocial Wellbeing of Sudanese Refugees: A Literature Review. Transcultural Psychiatry, 46 (2): 300-315.

Thapa, SB, Van Ommeren, M, Sharma, B, de Jong, JTVM, Hauff, E. (2003) Psychiatric disability among tortured Bhutanese refugees in Nepal. American Journal of Psychiatry, 160: 2032-37.

Vojvoda, D, Weine, SM, McGlashan, T, Becker DF, Southwick, SM. (2008) Posttraumatic stress disorder symptoms in Bosnian refugees $31 / 2$ years after resettlement. Journal of Rehabilitation Research \& Development, 45 (3): 421-26.

World Health Organization (WHO) (2010). Measuring Health and Disability: Manual for WHO Disability Assessment Schedule (WHODAS 2.0). Malta: World Health Organization (WHO). World Health Organization (WHO) (2002). Towards a Common Language for Functioning, Disability and Health: ICF. Geneva: World Health Organization.

World Health Organization (WHO) (1992). The ICD-10 Classification of Mental and Behavioural Disorders: Clinical Description and Diagnostic Guidelines. Geneva: World Health Organization. 